\title{
Difference between younger and older adults in post-task traces after creative task performance matches PASA model
}

\author{
Evgeniya Privodnova \\ Scientific Research Institute of \\ Physiology and Basic Medicine \\ Novosibirsk, Russian Federation \\ privodnovaeu@physiol.ru
}

\author{
Nina Volf \\ Scientific Research Institute of \\ Physiology and Basic Medicine \\ Novosibirsk, Russian Federation \\ volf@physiol.ru \\ Ekaterina Merculova \\ Scientific Research Institute of \\ Physiology and Basic Medicine \\ Novosibirsk, Russian Federation \\ merkaterine@gmail.com
}

\author{
Dariya Bazovkina \\ Scientific Research Institute of \\ Physiology and Basic Medicine \\ Novosibirsk, Russian Federation \\ daryabazovkina@gmail.com
}

\begin{abstract}
Brain activity during during execution of the experimental task in older adult differ from that in younger ones. HAROLD model describes hemispheric asymmetry reduction in older adults, while PASA model postulates posterior-anterior shift in aging. Up to date, age-related differences in use-dependent neuroplasticity are under-studied. Obtained results showed that in both pre-task and post-task intervals alpha power anteriorposterior gradient (posterior>anterior) was higher in younger group in comparison with older group. This pattern of age-related differences is consistent with PASA model.
\end{abstract}

Keywords - alpha rhythm, post-task traces, PASA, aging

\section{Motivation and aim}

\section{Motivation}

Repetitive cognitive activity induces neuroplasticity prosesses [1], which may manifests itself in electroencephalography (EEG) by post-task traces after task performance. It is well-known that brain activity during during execution of the experimental task in older adult differ from that in younger ones. Empirical data were summarized in several patterns of age-related changes probably reflecting neurocompensatory processes. HAROLD model describes hemispheric asymmetry reduction in older adults [2], while PASA model postulates posterior-anterior shift in aging [3]. Up to date, age-related differences in use-dependent neuroplasticity are under-studied.

Aim

The current study aimed to explore age-related differences in post-task traces after creative task performance and to examine whether these differences match the functional aging brain models (PASA, HAROLD).

\section{Methods}

We recorded EEG in 31 younger (19-33 years) and 30 older adults (55-75 years) before, during and after divergent thinking session. All subjects were students or continued their professional activity; exclusion criteria was self-reported history of psychiatric, major medical, and neurological diseases. Divergent thinking session lasted for 30-40 minutes and included 30 verbal tasks ("Alternate Uses Task", [4]) and 30 visual tasks ("Incomplete figures", [4]). The EEG data were registered using $52 \mathrm{Ag}-\mathrm{AgCl}$ electrodes placed according to the modified version of the international 10-20 system via "Neuroscan 4.4" (USA). Fronto-central electrode was used as the ground, and electronically linked mastoid electrodes as reference. Electrode impedances did not exceed $5 \mathrm{k} \Omega$. The EEG was amplified using Neuroscan amplifiers with a gain of 250 and a bandpass of $0-50 \mathrm{~Hz}$. Artifacts rejection was made using independent component analysis implemented in EEGLAB toolbox (https://sccn.ucsd.edu/eeglab/). Bandwidth for the upper alpha band was defined as [(individual alpha peak frequency) to (individual alpha peak frequency +2)]. To examine usedependent changes alpha activity, we used three-minute recordings in eyes-open resting-state condition in pre-test and post-test intervals. EEG data were segmented into $2 \mathrm{~s}$ epochs and submitted to further analysis.For each derivation, mean alpha power was calculated using Fourier transform via EEGLAB toolbox. Then derivations were combined in the frontal, central, temporal- central, and parietal-occipital left regions and the same right regions. For statictical analysis we used repeated measures analysis of variance (ANOVA) implemented in STATISTICA10.

\section{Results}

We found significant interaction of factors "region" and "time" $(\mathrm{F}(3,177)=46, \mathrm{p}<0.0001)$. For both age groups, alpha power in post-task interval was higher than at pre-task interval in all regions except of frontal (anterior-posterior gradient, posterior $>$ anterior). ANOVA yielded significant interaction of factors "region" and "age" $(\mathrm{F}(3,177)=3, \mathrm{p}<0.02)$. In younger group, alpha power in central and parietal-occipital region was higher than in frontal and temporal-central regions. In older group, alpha power at central brain region was higher in comparison with all the rest regions, however there was no difference between frontal and parietal-occipital regions. In both pre-task and post-task intervals alpha power anterior-posterior gradient (posterior $>$ anterior) was higher in younger group in comparison with older group $(\mathrm{p}<0.05)$. This pattern of agerelated differences is consistent with PASA model [3].

\section{ACKNOWLEDGMENT}

Supported by budgetary funding for basic scientific research (theme No. AAAA-A16-116021010228-0, EEG data collection) and by RFBR and Government of the Novosibirsk region according to the research project No. 19-415-543009 (EEG data processing and analysis, conceptualization, paper writing and preparation). 


\section{REFERENCES}

[1] P.Voss, M.E. Thomas, J.M. Cisneros-Franco, and E. de Villers-Sidani, "Dynamic Brains and the Changing Rules of Neuroplasticity: Implications for Learning and Recovery", Front. Psychol., vol. 8:e1657, Oct. 2017.

[2] B.M. Duda, M.M. Owens, E.S. Hallowell, and L.H. Sweet, "Neurocompensatory Effects of the Default Network in Older Adults", Front. Aging Neurosci., vol. 11:e111, Jun. 2019.
[3] S.W. Davis, N.A. Dennis, S.M. Daselaar, M.S. Fleck, and R. Cabeza, "Que PASA? The posterior-anterior shift in aging", Cereb. Cortex, vol. 18(5), pp. 1201-1209, May 2008.

[4] E.P. Torrance, "Torrance tests of creative thinking norms-technical manual : verbal tests, forms A and B : figural tests, forms A and B", Princeton, New Jersey Personal Press, 1966 